\title{
Select One
}

National Cancer Institute

\section{Source}

National Cancer Institute. Select One. NCI Thesaurus. Code C157109.

A directive to choose one of the options provided. 